\title{
Análise de Copolímeros de Etileno $\alpha$-Olefinas por meio de Técnicas de Fracionamento
}

\author{
Fernanda F. N. Escher, Luciana P. Silva e Griselda B. Galland \\ Instituto de Química, Universidade Federal do Rio Grande do Sul
}

Adriana Benetti

Ipiranga Petroquímica S.A., Triunfo, RS

\begin{abstract}
Resumo: Este trabalho apresenta a avaliação da heterogeneidade de resinas comerciais de copolímeros de etileno-1-buteno, produzidas por diferentes processos com catalisadores Ziegler-Natta e de resinas de copolímeros de etileno-1-hexeno sintetizadas em laboratório com catalisadores metalocênicos suportados e em solução. Para fazer a avaliação da heterogeneidade foram empregadas técnicas de fracionamento por extração com diferentes solventes e temperaturas e fracionamento térmico por DSC. As frações obtidas foram caracterizadas por ${ }^{13} \mathrm{C}-\mathrm{NMR}$, FTIR, SEC e DSC. Como esperado, foi confirmada uma maior heterogeneidade das resinas sintetizadas com catalisador Ziegler-Natta quando comparadas às resinas metalocênicas. A resina obtida com catalisador Ziegler-Natta, produzida pelo processo Union Carbide (UCC), apresenta uma incorporação mais homogênea de comonômero do que o processo Spherilene. A resina obtida com catalisador metalocênico homogêneo permite maior incorporação de comonômero resultando em uma maior heterogeneidade no tamanho das cadeias comparada com a obtida com catalisador metalocênico suportado.
\end{abstract}

Palavras-chave: Copolímeros de etileno, microestrutura, fracionamento, ${ }^{13} \mathrm{C}-\mathrm{NMR}, \mathrm{FTIR}, \mathrm{SEC}$ e DSC.

\section{Ethylene Copolymers Analysis by Fractionation Techniques}

Abstract: Samples of ethylene 1-butene copolymer, produced in laboratory by different processes using ZieglerNatta catalysts, ethylene-1-hexene and metallocene catalysts, were fractionated according to molecular size by direct extraction by different solvents and at different temperatures. These samples were also fractionated by differences on crystallinity using a DSC calorimeter. The obtained fractions were characterized by ${ }^{13} \mathrm{C}$ NMR , FTIR, GPC and DSC. The polyethylene copolymers produced by Ziegler-Natta catalyst were found more heterogeneous when compared with the one produced by metallocene catalysts. The commercial resin produced by UCC process with Ziegler-Natta catalysts showed a more homogeneous incorporation of comonomer compared with the one obtained by the Spherilene process. The supported metallocene catalyst gave a more homogeneous product than the homogeneous metallocene one.

Keywords: Ethylene copolymers, microstructure, fractionation, ${ }^{13} \mathrm{C}-\mathrm{NMR}, \mathrm{DSC}, \mathrm{SEC}, \mathrm{FTIR}$.

\section{Introdução}

O fracionamento é o método mais utilizado para avaliar a heterogeneidade da estrutura molecular do polietileno linear de baixa densidade (PELBD). Muitos trabalhos utilizam a técnica de fracionamento por eluição com gradiente de temperatura (TREF) ou mais recentemente o fracionamento por cristalização (CRYSTAF) ${ }^{[1-3]}$. Nas técnicas de TREF e CRYSTAF a separação em diferentes frações está baseada na diferença de cristalinidade dos segmentos da cadeia polimérica devido à composição química variável.

Autora para correspondência: Griselda B. Galland, Instituto de Química, Universidade Federal do Rio Grande do Sul, Av. Bento Gonçalves 9500, CEP: 91501-970, Porto Alegre, RS. E-mail: griselda@if.ufgs.br 
Como ainda não dispomos dessa técnica, fizemos a avaliação da heterogeneidade estrutural das resinas de PELBD utilizando o fracionamento por extração com diferentes solventes e temperaturas e o fracionamento térmico por DSC.

No fracionamento por extração ${ }^{[4]} \mathrm{o}$ princípio de separação é governado pelo grau de solubilidade de cada espécie individual do polímero. Esta separação é feita em etapas utilizando solventes e temperaturas diferentes. Assim, devido à solubilidade diferente das espécies, o polímero é subdividido em frações as quais apresentam diferentes massas molares e distribuições de massas molares. Esse tipo de fracionamento apresenta a vantagem de empregar equipamentos simples, pequenas quantidades de solvente e uso direto da amostra.

O fracionamento térmico ${ }^{[5]}$ por calorimetria diferencial de varredura (DSC) é uma técnica baseada no princípio de separação por diferença de cristalinidade, assim como o TREF e o CRYSTAF. Esse método consiste em uma etapa de cristalização da cadeia de polietileno por meio de sucessivos annealing, em temperaturas decrescentes a partir da temperatura de fusão do polímero, e posterior reaquecimento novamente até a fusão.

A heterogeneidade estrutural de um polímero é devida ao modo de inserção de comonômero à cadeia polimérica $^{[6-8]}$. Dependendo do sistema catalítico e das condições de polimerização pode ocorrer a formação de cadeias poliméricas com diferentes tamanhos e teor de incorporação de comonômero. Desta forma a heterogeneidade composicional pode ser analisada de duas maneiras:

- intramolecular onde é analisada a distribuição das ramificações provenientes da incorporação do comonômero ao longo da cadeia.

- intermolecular onde são analisadas as diversas cadeias com composição equivalente.

Assim para conhecermos melhor as características de copolímeros de etileno buscamos neste trabalho, por meio das técnicas de fracionamento por extração com diferentes solventes e temperaturas e fracionamento térmico, fazer uma avaliação da heterogeneidade das resinas obtidas por diferentes processos de polimerização.

Quatro resinas foram analisadas, duas resinas comerciais A e B obtidas com catalisadores ZieglerNatta por diferentes processos o Spherilene e o Union
Carbide (UCC), respectivamente, e duas resinas, sintetizadas com catalisadores metalocênicos, de copolímeros de etileno-1-hexeno identificadas por C e D. As resinas A e B são copolímeros de etileno1-buteno com densidade de 0,924 e $0,923 \mathrm{~g} / \mathrm{cm}^{3}$, respectivamente. A resina $\mathrm{C}$ foi obtida utilizando o catalisador metalocênico $(\mathrm{nBuCp})_{2} \mathrm{ZrCl}_{2}$ suportado em MAO e sílica e a resina D sintetizada com o mesmo catalisador porém em solução. ${ }^{[9]}$ As frações obtidas foram caracterizadas por DSC, GEC, FTIR, ${ }^{13} \mathrm{C}-\mathrm{NMR}$.

\section{Experimental}

\section{Fracionamento por extração com diferentes solventes e temperaturas}

Foi realizado o fracionamento das resinas de PELBD em extrator Soxhlet. Foram utilizados os seguintes solventes: éter etílico $\left(34,5^{\circ} \mathrm{C}\right)$; n-hexano $\left(68,7^{\circ} \mathrm{C}\right)$; n-heptano $\left(98,4^{\circ} \mathrm{C}\right)$ e tolueno $\left(111,0^{\circ} \mathrm{C}\right)$.

Para cada fracionamento foram utilizados cerca de $5 \mathrm{~g}$ de amostra moída. O polímero foi colocado em cartuchos de papel próprios para extração. Cada solvente permaneceu circulando sobre a amostra por 16 horas. Para garantir um fracionamento eficiente foram necessários $400 \mathrm{~mL}$ de cada solvente (Esquema 1). As frações obtidas foram coletadas, na temperatura ambiente, precipitadas em etanol, filtradas e transferidas para pratos de papel de alumínio os quais permaneceram em chapas sob aquecimento até que todo o solvente fosse evaporado. Em seguida as amos-

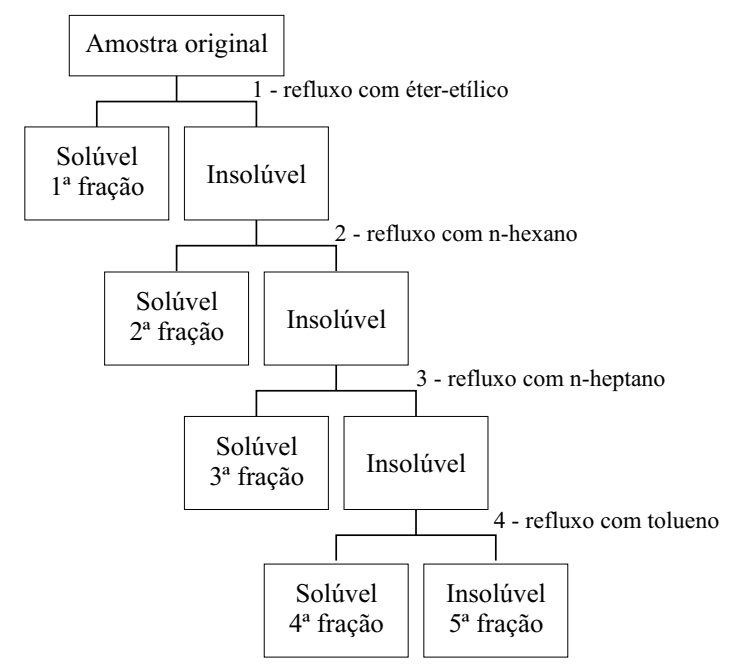

Esquema 1. Fracionamento por extração com diferentes solventes e diferentes temperaturas. 
tras foram colocadas em dissecador com sílica até atingir massa constante (5 a 7 dias). Para cada amostra foram realizadas três repetições.

\section{Análise Térmica:}

A análise térmica das amostras foi realizada em um DSC Thermal Analyst 2100, com resfriamento automático, de duas maneiras:

Método 1 - o mais comum usado na análise de polímeros semi-cristalinos - Foram pesados de 4 - 10 $\mathrm{mg}$ de cada fração obtida no fracionamento por solvente. A amostra foi aquecida até $160^{\circ} \mathrm{C}$ a uma taxa de $20^{\circ} \mathrm{C} /$ min e então resfriada até $20^{\circ} \mathrm{C}$ em uma taxa de $10^{\circ} \mathrm{C} / \mathrm{min}$. Essa temperatura foi mantida por $5 \mathrm{mi}-$ nutos e a amostra foi reaquecida a $10^{\circ} \mathrm{C} / \mathrm{min}$ até $160^{\circ} \mathrm{C}$.

Método 2 - "Fracionamento térmico" (somente para as amostras sintetizadas com catalisador ZieglerNatta). Foram pesados 4-10 mg de amostra sendo esta aquecida até $160^{\circ} \mathrm{C}$ a uma taxa de $20^{\circ} \mathrm{C} / \mathrm{min}$. Em seguida foi submetida a sucessivos annealing (sob nitrogênio) com temperaturas decrescendo a partir da fusão. Foram utilizadas as seguintes temperaturas para o annealing $120,105,90,75$ e $50^{\circ} \mathrm{C}$. O tempo de cada etapa de annealing foi de $2 \mathrm{~h}$. Após o resfriamento a amostra foi reaquecida a $10^{\circ} \mathrm{C} / \mathrm{min}$ até $160^{\circ} \mathrm{C}$. Devido ao longo tempo de utilização do equipamento utilizado para esta análise (cerca de 14 horas para cada análise) optou-se por fazer somente o fracionamento térmico das amostras originais e de suas frações insolúveis.

\section{Cromatografia de exclusão por tamanho, SEC}

As análises foram realizadas em um aparelho Waters modelo 150C, equipado com detector de índice de refração diferencial tipo deflexão ótica, utilizando fibras óticas. Foram utilizadas três colunas com partículas de $10 \mathrm{~mm}$, com poros de $10^{6}, 10^{4}$ e $500 \AA$ respectivamente. A análise foi realizada utilizando 1,2,4-triclorobenzeno (TCB) como solvente, com fluxo de $1,0 \mathrm{~mL} / \mathrm{min}$ a temperatura de $145^{\circ} \mathrm{C}$ durante uma hora para assegurar total eluição da amostra. As amostras foram dissolvidas em uma concentração de $0,2 \%$, em TCB (foram deixadas na estufa por cerca de 30 min para total solubilização da amostra). A curva de calibração universal foi obtida utilizando 20 padrões de poliestireno, 3 de polietileno e 5 de polipropileno. Os principais parâmetros determinados foram: $\overline{\mathrm{M}} \mathrm{w}$ - massa molar ponderal média, $\overline{\mathrm{M}} \mathrm{n}$ massa molar numérico média; $\overline{\mathrm{M}} \mathrm{w} / \overline{\mathrm{M}} \mathrm{n}$ - distribuição de massas molares ou polidispersão.

\section{Espectroscopia no Infravermelho com Transformada de Fourier, FTIR}

As amostras originais e suas frações foram prensadas entre folhas de alumínio a $150^{\circ} \mathrm{C}$, sob carga de 5 a 4 ton, por 30 s. Os filmes foram analisados em espectrômetro de infravermelho com Transformada de Fourier (FTIR) Nicolet, modelo Magna 560, com resolução nominal de $2 \mathrm{~cm}^{-1}$. Os espectros de infravermelho gerados abrangem a região de 4800 a $400 \mathrm{~cm}^{-1}$.

Neste trabalho foi analisada a banda de metilas $\left(1378 \mathrm{~cm}^{-1}\right)$, uma vez que para quantificar a banda de ramificação específica $\left(770 \mathrm{~cm}^{-1}\right)$ seria necessário um filme de maior espessura (o que em algumas amostras não era possível). Para a quantificação do teor (em massa) de comonômero incorporado foi desenvolvido um método de cálculo no equipamento Nicolet utilizando o programa Gramns onde a absorbância a $1378 \mathrm{~cm}^{-1}$, após subtração espectral de um padrão de UHMPE, foi relacionada com a área da região compreendida entre 4482 e $3950 \mathrm{~cm}^{-1}[7]$. Para a construção das curvas de calibração foram utilizados padrões de $\mathrm{C}_{2} / \mathrm{C}_{4}$ fornecidos pela IPQ e $\mathrm{C}_{2} / \mathrm{C}_{6}$ obtidos no IQ da UFRGS. Com o auxílio dessas curvas foram determinados os teores de incorporação de $\mathrm{C}_{4}$ e de $\mathrm{C}_{6}$, respectivamente, assim como o número de ramificações por $1000 \mathrm{C}\left(\mathrm{CH}_{3} / 1000 \mathrm{C}\right)$ nas amostras originais e em suas frações.

Espectroscopia de ressonância magnética nuclear de carbono $13,{ }^{13} \mathrm{C}-\mathrm{NMR}$

As amostras para análise de ${ }^{13} \mathrm{C}$-NMR foram preparadas dissolvendo $0,05 \mathrm{~g}$ de polímero em aproximadamente $0,4 \mathrm{~mL}$ de triclorobenzeno, a $120^{\circ} \mathrm{C}$ com agitação freqüente para a solubilização completa. Em seguida, foi adicionado $0,1 \mathrm{~mL}$ de benzeno deuterado. O equipamento utilizado foi o Varian, modelo Inova300, operando a $75 \mathrm{MHz}$ empregando um ângulo de pulso de $74^{\circ}$, tempo de aquisição de $1,5 \mathrm{~s}$, tempo de relaxação de $4 \mathrm{~s}$ e a $120^{\circ} \mathrm{C}$. Foram obtidos os espectros de ${ }^{13} \mathrm{C}$ desacoplados dos ${ }^{1} \mathrm{H}$. O número de ramificações por $1000 \mathrm{C}\left(\mathrm{CH}_{3} / 1000 \mathrm{C}\right)$ foi determinado da seguinte maneira:

- percentual de comonômero incorporado:

Em mol:

$\% \mathrm{C}=([\mathrm{C}] \times 100) /([\mathrm{E}]+[\mathrm{C}])$ 
Em massa:

$\% \mathrm{C}=([\mathrm{C}] \times \mathrm{M}$ de $\mathrm{C} \times 100) /([\mathrm{E}] \times \mathrm{M}$ de $\mathrm{E}+[\mathrm{C}] \times \mathrm{M}$ de $\mathrm{C})$ onde:

M: massa molar;

$[\mathrm{C}]=[\mathrm{CCC}]+[\mathrm{ECC}]+[\mathrm{ECE}]$;

[ C ] = fração molar de comonômero;

$[\mathrm{E}]=[\mathrm{EEE}]+[\mathrm{CEC}]+[\mathrm{CEE}]$

$[E]=$ fração molar de etileno

$\mathrm{O}$ resultado do teor de comonômero incorporado pode ser expresso em número de ramificações/ 1000C:

$\mathrm{CH}_{3} / 1000 \mathrm{C}=(\% \mathrm{~mol} \mathrm{C} \times 1000) / 200$

\section{Resultados e Discussão}

Na Tabela 1 estão relacionados os resultados do fracionamento por extração e a análise das frações obtidas. No procedimento de extração por diferen- tes solventes, em diferentes temperaturas, foi possível obter um perfil do diagrama de distribuição de composição química (DCQ) da amostra por meio do percentual de massa extraída em cada solvente a diferentes temperaturas (Figura 1). Na Figura 1 observa-se que a amostra A sofreu uma maior extração (\% em massa) quando em presença de n-hexano e n-heptano. Nestes solventes foram extraídas as cadeias de massa molar (Mw) variando entre 20.000 e 30.000 com um teor de comonômero incorporado de 10 e $8 \%$ em massa, respectivamente. Já para a amostra B, a extração mais eficiente (maior \% em massa) foi obtida em presença de tolueno onde, foram extraídas as cadeias de elevadas massas molares (Mw 136.000) com 4,7\% em massa de comonômero incorporado.

Entretanto, para as amostras C e D, sintetizadas com catalisadores metalocênicos, obteve-se somente uma fração solúvel em tolueno. Isso mostra que as

Tabela 1. Caracterização das amostras fracionadas

Cronômetro incorporado

\begin{tabular}{|c|c|c|c|c|c|c|c|c|c|c|c|c|}
\hline Amostra & Massa d & fração & $\overline{\mathbf{M}} \mathbf{w}$ & $\overline{\mathbf{M n}} \mathbf{n}$ & $\overline{\mathbf{M}} \mathbf{w} / \overline{\mathbf{M}} \mathbf{n}$ & $\mathbf{T m}$ & Crist. & & TIR & & ${ }^{13} \mathrm{C}-\mathrm{N}$ & \\
\hline (1) & (mg) & $(\%)$ & $(\mathrm{g} / \mathrm{mol})$ & $(\mathrm{g} / \mathrm{mol})$ & & $\left({ }^{\circ} \mathrm{C}\right)^{(2)}$ & $\%^{(2)}$ & $(\% \mathrm{w})$ & $\mathrm{CH}_{3} / 1000 \mathrm{C}$ & $(\% \mathrm{~mol})$ & (\%w) & $\mathrm{CH}_{3} / 1000 \mathrm{C}$ \\
\hline $\mathrm{AF} 1$ & 78,900 & 1,60 & 10500 & 1600 & 6,6 & 66 & 5,00 & 10,7 & 28,4 & - & - & - \\
\hline $\mathrm{AF} 2$ & 164,90 & 3,30 & 21200 & 5100 & 4,2 & 82 & 14,0 & 9,9 & 26,2 & - & - & - \\
\hline $\mathrm{AF} 3$ & 140,00 & 2,80 & 30800 & 6600 & 4,7 & 102 & 21,6 & 8,0 & 21,1 & - & - & - \\
\hline $\mathrm{AF} 4$ & 74,800 & 1,50 & 59200 & 17100 & 3,5 & 114 & 27,5 & 6,3 & 16,3 & - & - & - \\
\hline AF5 & 4540,6 & 90,6 & 121700 & 22400 & 5,4 & 127 & 54,0 & 5,3 & 13,6 & 3,7 & 7,1 & 18,4 \\
\hline A & - & - & 135200 & 34400 & 3,9 & 127 & 54,2 & 6,1 & 15,9 & 3,8 & 7,3 & 19,1 \\
\hline $\mathrm{BF} 1$ & 44,800 & 0,80 & 107000 & 21100 & 5,1 & 120 & 48,3 & 5,9 & 15,2 & - & - & - \\
\hline $\mathrm{BF} 2$ & 56,600 & 1,10 & 118300 & 18500 & 6,4 & 121 & 53,9 & 5,5 & 14,1 & - & - & - \\
\hline $\mathrm{BF} 3$ & 58,900 & 1,10 & 127300 & 19400 & 6,6 & 121 & 51,6 & 5,4 & 14,0 & - & - & - \\
\hline $\mathrm{BF} 4$ & 462,80 & 9,00 & 136500 & 33400 & 4,1 & 122 & 52,1 & 4,7 & 11,9 & - & - & - \\
\hline BF5 & 4308,1 & 84,3 & 144000 & 35800 & 4,0 & 122 & 53,2 & 4,2 & 10,8 & 3,1 & 6,1 & 15,7 \\
\hline B & - & - & 151000 & 32000 & 4,7 & 122 & 53,6 & 4,5 & 11,3 & 3,3 & 6,3 & 16,2 \\
\hline CF4 & 137,67 & 27,2 & 33100 & 17300 & 1,9 & 104,4 & 25,0 & 11,8 & 22,4 & 3,9 & 10,8 & 19,5 \\
\hline CF5 & 3363,8 & 66,5 & 34100 & 19000 & 1,8 & 104,5 & 26,5 & 11,5 & 21,6 & 3,8 & 10,6 & 19,1 \\
\hline $\mathrm{C}$ & - & - & 35900 & 18700 & 1,9 & 104,8 & 25,6 & 11,2 & 21,1 & 4,1 & 11,4 & 20,5 \\
\hline DF4 & 363,22 & 37,3 & 7400 & 4000 & 1,8 & 96,0 & 29,7 & $*$ & * & 6,5 & 17,2 & 32,5 \\
\hline DF5 & 2232,6 & 44,1 & 15600 & 4300 & 3,6 & 119,0 & 41,8 & * & $*$ & 4,2 & 11,6 & 21,0 \\
\hline D & - & - & 18900 & 4400 & 4,2 & 116,9 & 26,7 & * & * & 3,9 & 10,8 & 19,5 \\
\hline
\end{tabular}

1- AF1: amostra A fração 1, etc...; BF1: amostra B fração 1, etc....

2- Tm, e \% crist. - método A.

(*) Não foram realizadas as análises de FTIR para a amostra D, pois não foi possível obter filmes em condições de serem analisados nas mesmas condições de prensagem das outras amostras. 


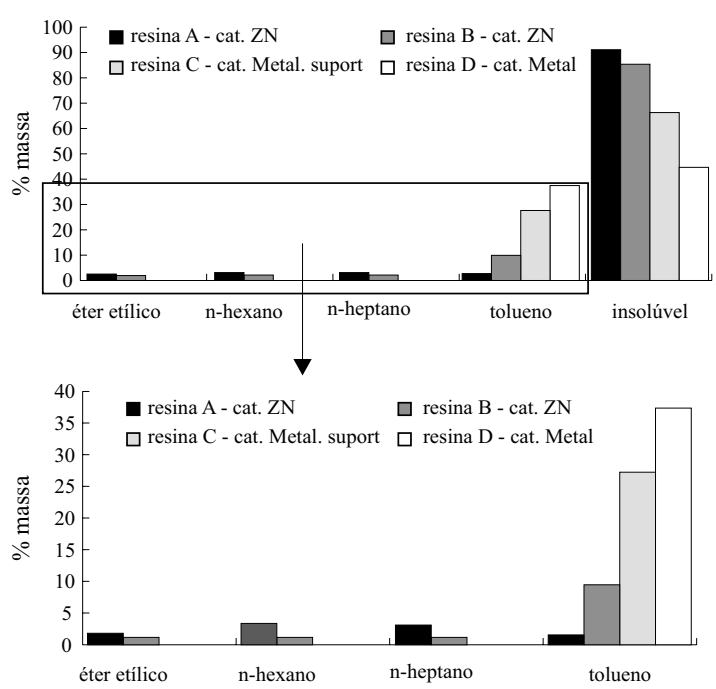

Figura 1. Distribuição de composição química (DCQ) obtida por extração com diferentes solventes em diferentes temperaturas.

resinas metalocênicas apresentam cadeias mais uniformes quando comparadas às resinas Ziegler-Natta.

As análises por SEC das frações revelaram que a amostra A apresenta maior heterogeneidade no tamanho das cadeias, enquanto que a amostra B apresenta apenas pequenas variações de massa molar (Figura 2). Para as resinas metalocênicas observa-se que a amostra C, sintetizada com catalisador suportado, apresenta maiores massas molares que a amostra $\mathrm{D}$ e praticamente o mesmo tamanho de cadeia, enquanto que a amostra $\mathrm{D}$, sintetizada com o catalisador metalocênico solúvel, apresenta variações no tamanho de cadeia.

Pela relação entre o Mw (obtido por SEC) e o teor de incorporação (obtido por FTIR para as resinas Ziegler-Natta e por ${ }^{13} \mathrm{C}$ NMR para as resinas metalocênicas) verificou-se que os copolímeros mais ramificados apresentam tamanho médio de cadeias menores (Figura 3). Consequentemente, devido à formação de cristais de tamanhos menores e mais imperfeitos houve uma redução da temperatura de fusão (Figura 4) e da cristalinidade (Tabela 1). Esses com-

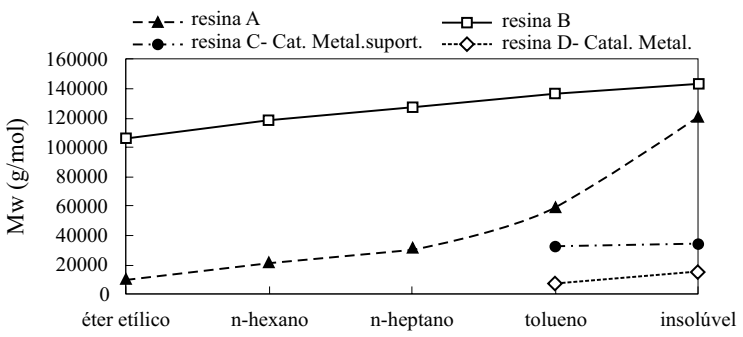

Figura 2. Variação do $\overline{\mathrm{Mw}}$ em cada fração

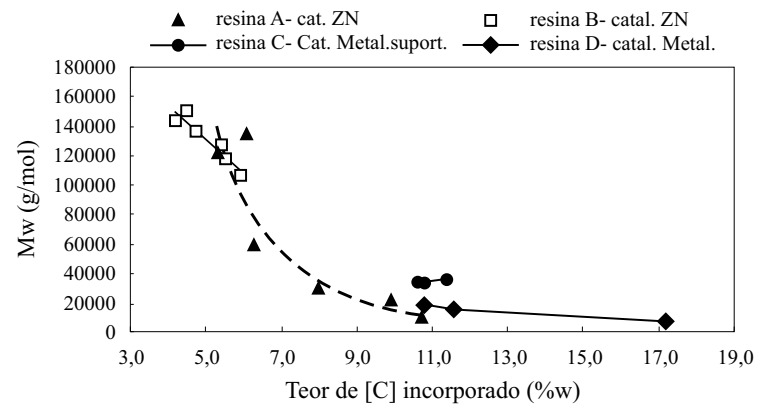

Figura 3. Influência do teor de comonômero na massa molar de cada fração.

portamentos foram verificados para as duas resinas Ziegler-Natta (A e B), entretanto, como já mencionado anteriormente, devido à maior heterogeneidade das cadeias da amostra A estas variações foram mais expressivas.

A amostra sintetizada com catalisador metalocênico homogêneo (D) apresenta uma heterogeneidade tanto no tamanho das cadeias como na distribuição de comonômero (Figura 3). A fração solúvel em tolueno apresenta cadeias com elevado teor de comonômero incorporado e consequentemente menor peso molecular do que a fração insolúvel e a amostra bruta. A análise da amostra $\mathrm{C}$ (catalisador suportado) revelou que estas frações são bastante homogêneas apresentam praticamente o mesmo tamanho de cadeia e teor de incorporação semelhantes.

A maior incorporação de comonômero na cadeia resulta em uma redução significativa da temperatura de fusão da fração solúvel em tolueno da amostra D (Figura 4).

A análise de ${ }^{13} \mathrm{C}$-NMR (Tabela 1) das resinas brutas obtidas com catalisador Ziegler-Natta mostraram que estas apresentam praticamente o mesmo teor de comonômero, entretanto por DSC constatou-se que a resina $\mathrm{B}$ apresenta uma temperatura de fusão inferior quando comparada com a resina A. Isso indica que na resina $\mathrm{B}$ os segmentos de etileno, separados por

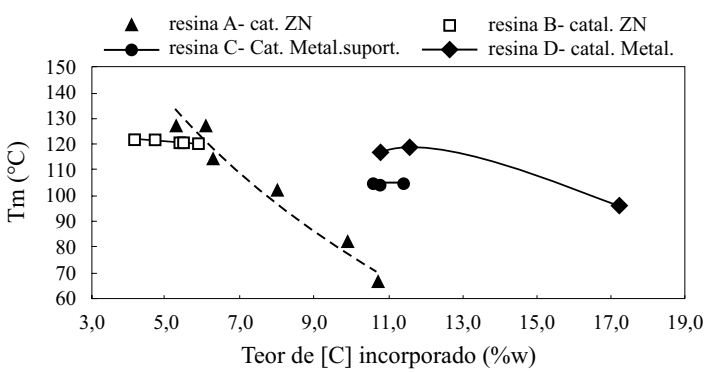

Figura 4. Influência do teor de comonômero na temperatura de fusão de cada fração. 


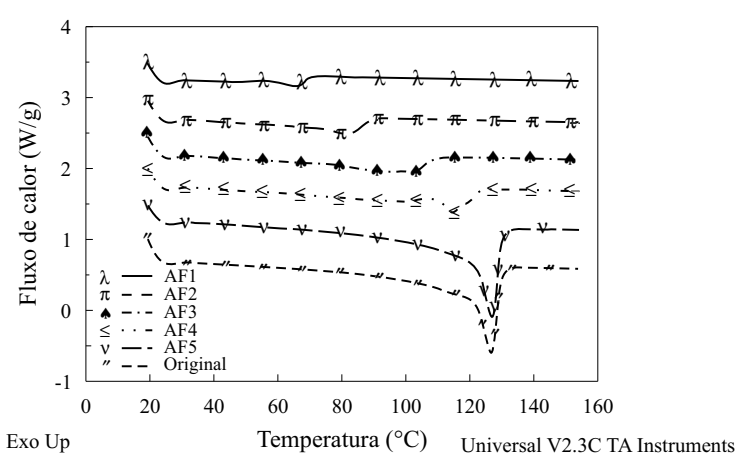

Figura 5. Sobreposições das curvas de fusão da amostra A e de suas frações.

ramificações, apresentam extensões semelhantes formando cristais de tamanho menores, favorecendo a redução da temperatura de fusão. Para a resina $\mathrm{A}$, no entanto, esses segmentos de etileno, separados por ramificações, apresentam tamanhos diferentes (uns maiores, outros menores) fazendo com que a temperatura de fusão não reduza tanto quanto a da resina $\mathrm{B}$ para um mesmo teor de comonômero.

A observação dos conjuntos de curvas de DSC (segundo o método 1 descrito no procedimento experimental) das frações da amostra A (Figura 5) indica uma distribuição bastante heterogênea e que quanto maior a concentração molar de comonômero, maior é a largura da endoterma de fusão, a tal ponto que a identificação de um pico máximo de temperatura torna-se menos precisa em frações com elevado teor de comonômero incorporado.

A largura da endoterma expressa a variedade de tamanho das lamelas formadas durante a cristalização a partir do fundido. As frações mais modificadas possuem lamelas menos espessas, as quais fundem em temperaturas inferiores. Assim, quanto maior o grau de modificação médio da fração, menor será a temperatura inicial da endoterma de fusão e mais larga esta será.

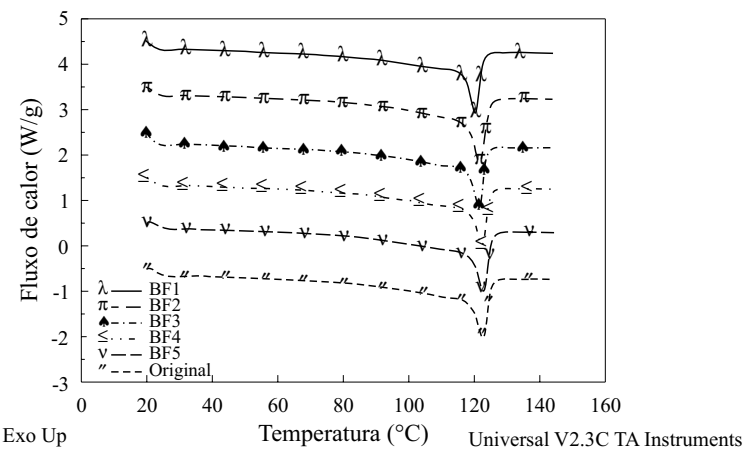

Figura 6. Sobreposição das curvas de fusão da amostra B e de suas frações.

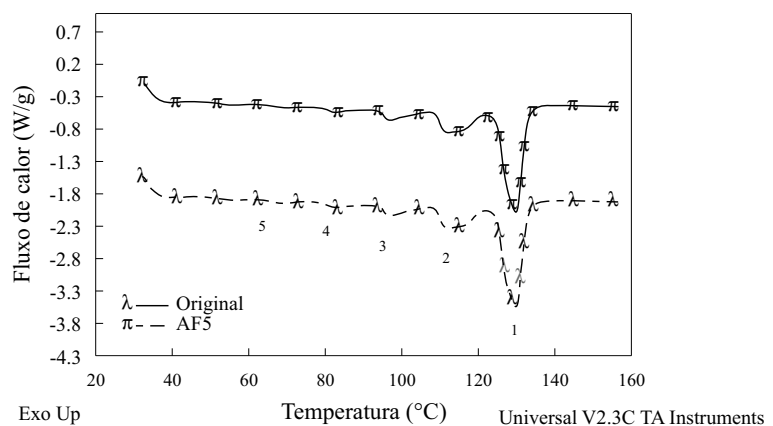

Figura 7. Curvas de fusão da amostra A e sua fração insolúvel após o fracionamento térmico por DSC (método 2).

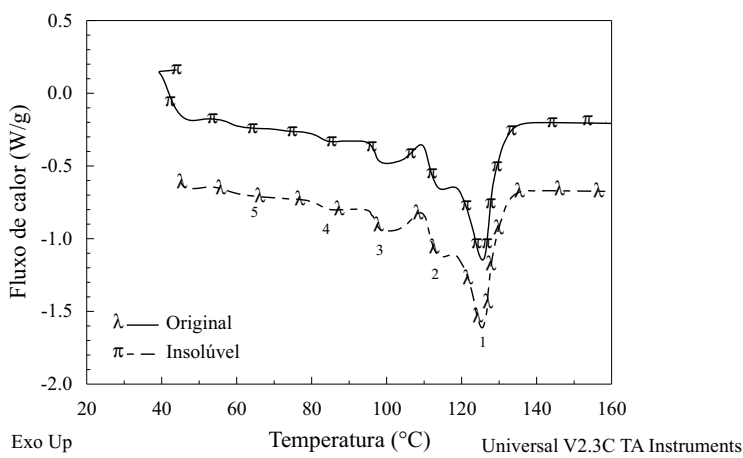

Figura 8. Curvas de fusão da amostra $\mathrm{B}$ e sua fração insolúvel após o fracionamento térmico por DSC (método 2).

Para a amostra B, o conjunto de curvas de DSC (Figura 6) mostra que as curvas das frações se assemelham na forma, mesmo que seus picos máximos de fusão tenham um pequeno deslocamento, conforme o teor de comonômero incorporado na cadeia. É possível verificar que não há cadeias com elevados graus de modificação, nem cadeias praticamente isentas de ramificações.

O polímero não fracionado e sua correspondente fração insolúvel foram termicamente tratadas ("fracionamento térmico"- método 2). A seguir são apresentadas as curvas de DSC após o fracionamento térmico para as duas resinas (Figura 7 e 8).

Como pode ser visto a curva de DSC do copolímero é resolvido em uma série de cinco picos. As curvas apresentam a distribuição composicional das amostras, onde o perfil produzido é o resultado da distribuição multimodal de lamelas cristalinas de diferentes espessuras, em função dos graus de modificação da cadeias.

Embora seja observada uma sobreposição de picos para duas frações poliméricas sucessivas, a endoterma difere de uma fração para outra. Há uma redução na variação da intensidade do pico das frações insolúveis. Isso indica que foram extraídos segmentos cristalizáveis por meio da extração com solvente (Tabela 2) .

Polímeros: Ciência e Tecnologia, vol. 10, nº 4, p. 202-208, 2000 
Tabela 2. Análises das curvas de fusão das amostras submetidas ao fracionamento térmico por DSC

\begin{tabular}{cccccc}
\hline \multicolumn{4}{c}{ Amostra A - Spherilene } \\
\hline \multicolumn{4}{c}{ Original } & \multicolumn{2}{c}{ Insolúvel } \\
Pico & $\mathrm{Tm}$ & $\Delta \mathrm{H}$ & Crist. & $\Delta \mathrm{H}$ & Crist. \\
& $\left({ }^{\circ} \mathrm{C}\right)$ & $(\mathrm{J} / \mathrm{g})$ & $(\%)$ & $(\mathrm{J} / \mathrm{g})$ & $(\%)$ \\
\hline 1 & 127 & 49,7 & 17,1 & 43,8 & 15,1 \\
2 & 111 & 15,6 & 5,4 & 14,1 & 4,8 \\
3 & 96 & 6,0 & 2,0 & 5,4 & 1,8 \\
4 & 82 & 2,3 & 0,8 & 1,8 & 0,6 \\
5 & 59 & 2,5 & 0,8 & 1,3 & 0,4 \\
\hline
\end{tabular}

\begin{tabular}{cccccc}
\hline \multicolumn{5}{c}{ Amostra B - UCC } \\
\hline \multirow{4}{*}{ Pico } & Tm & $\Delta \mathrm{H}$ & Crist. & $\Delta \mathrm{H}$ & Crist. \\
& $\left({ }^{\circ} \mathrm{C}\right)$ & $(\mathrm{J} / \mathrm{g})$ & $(\%)$ & $(\mathrm{J} / \mathrm{g})$ & $(\%)$ \\
\hline 1 & 122 & 54,2 & 18,6 & 51,6 & 17,8 \\
2 & - & - & - & - & - \\
3 & 99 & 7,5 & 2,6 & 6,8 & 2,3 \\
4 & 84 & 1,4 & 0,5 & 1,1 & 0,4 \\
5 & 62 & 1,0 & 0,3 & 0,8 & 0,3 \\
\hline
\end{tabular}

\section{Conclusões}

A técnica de fracionamento por extração com diferentes solventes e temperaturas foi eficiente na separação de cada espécie individual de copolímeros de etileno servindo como uma ferramenta importante para a caracterização dos mesmos. Esta técnica apresenta a vantagem de empregar equipamentos simples com pequenas quantidades de solventes, entretanto produz um visão "grosseira" da heterogeneidade da amostra uma vez que as faixas de temperaturas de extração são amplas $\left(20\right.$ a $\left.30^{\circ} \mathrm{C}\right)$.

Observa-se que a separação da amostra em frações foi devido a diferença de solubilidade das espécies, a qual é influenciada pela massa molar e pela cristalinidade das diferentes cadeias que constituem o polímero.

O conjunto de resultados obtidos mostrou que as resinas Ziegler-Natta são bastante heterogêneas quando comparadas as resinas metalocênicas.

Comparando as resinas Ziegler-Natta observa-se que a resina $\mathrm{B}$, produzida pelo processo UCC apresenta uma incorporação mais homogênea de comonômero ao longo da cadeia do que a do processo Spherilene.
Para as resinas metalocênicas o sistema homogêneo produziu uma maior incorporação de comonômero na cadeia polimérica resultando em uma distribuição mais larga no tamanho das cadeias. Os copolímeros obtidos com o metaloceno suportado em MAO/sílica mostraram-se mais homogêneos, tanto em distribuição de comonômero, como em pesos moleculares.

O fracionamento térmico auxilia bastante na identificação de amostras com elevada heterogeneidade, entretanto para amostras mais homogêneas poucas informações podem ser obtidas. A desvantagem desta técnica é o longo tempo de uso do equipamento.

\section{Agradecimentos}

Os autores agradecem ao RHAE/CNPq pela concessão de uma bolsa e à IPIRANGA Petroquímica SA por ter colocado à disposição suas instalações.

\section{Referências Bibliográficas}

1. Monrabal, B. - New Trens in Polyolefins Science and Technology, p. 119 (1996).

2. Monrabal, B. - J. Appl. Polym. Sci., 52, p. 491 (1994).

3. Soares, J. B. P.; Hamielec, A. E. - Polymer, 36, p. 1639 (1995).

4. Frankuskiewics, F. - "Polymer Fractionation"; Springer-Verlag, (1994).

5. Adisson E.; Ribeiro, M.; Deffieux, A.; Fontanille, M. - Polymer, 33, p. 4337 (1992).

6. Soares, J. B. P.; Hamielec, A. E. - Polymer, 36, p. 2257 (1995).

7. Dal Pizzol, M. F. - “ Estudo das Estruturas de polietilenos lineares de baixa densidade obtidos com catalisadores Ziegler-Natta e metalocênicos" Dissertação de Mestrado Universidade Federal do Rio Grande do Sul, Brasil (1997).

8. Silva Filho, A. A. da - “ Caracterização Microestrutural de copolímeros de etileno com a-olefinas e blendas de reator", Dissertação de Mestrado Universidade Federal do Rio Grande do Sul, Brasil (1999).

9. Galland, G. B.; Seferin, M.; Mauler, R. S.; dos Santos, J. H. Z. - Polym. Int., 48, p.660 (1999).

Recebido: $13 / 07 / 00$

Aprovado: 13/11/00 